\title{
Barriers and facilitators to the implementation of a school-based physical activity policy in Canada: application of the theoretical domains framework
}

\author{
Katie A. Weatherson', Rhyann McKay², Heather L. Gainforth² and Mary E. Jung ${ }^{3 *}$
}

\begin{abstract}
Background: In British Columbia Canada, a Daily Physical Activity (DPA) policy was mandated that requires elementary school teachers to provide students with opportunities to achieve 30 min of physical activity during the school day. However, the implementation of school-based physical activity policies is influenced by many factors. A theoretical examination of the factors that impede and enhance teachers' implementation of physical activity policies is necessary in order to develop strategies to improve policy practice and achieve desired outcomes. This study used the Theoretical Domains Framework (TDF) to understand teachers' barriers and facilitators to the implementation of the DPA policy in one school district. Additionally, barriers and facilitators were examined and compared according to how the teacher implemented the DPA policy during the instructional school day.
\end{abstract}

Methods: Interviews were conducted with thirteen teachers and transcribed verbatim. One researcher performed barrier and facilitator extraction, with double extraction occurring across a third of the interview transcripts by a second researcher. A deductive and inductive analytical approach in a two-stage process was employed whereby barriers and facilitators were deductively coded using TDF domains (content analysis) and analyzed for sub-themes within each domain. Two researchers performed coding.

Results: A total of 832 items were extracted from the interview transcripts. Some items were coded into multiple TDF domains, resulting in a total of 1422 observations. The most commonly coded TDF domains accounting for $75 \%$ of the total were Environmental context and resources $(E C R ; n=250)$, Beliefs about consequences $(n=225)$, Social influences $(n=193)$, Knowledge $(n=100)$, and Intentions $(n=88)$. Teachers who implemented DPA during instructional time differed from those who relied on non-instructional time in relation to Goals, Behavioural regulation, Social/professional role and identity, Beliefs about Consequences. Forty-one qualitative sub-themes were identified across the fourteen domains and exemplary quotes were highlighted.

Conclusions: Teachers identified barriers and facilitators relating to all TDF domains, with ECR, Beliefs about consequences, Social influences, Knowledge and Intentions being the most often discussed influencers of DPA policy implementation. Use of the TDF to understand the implementation factors can assist with the systematic development of future interventions to improve implementation.

Keywords: School, Physical activity, Policy, Implementation, Barriers, Facilitators, Theoretical domains framework

\footnotetext{
* Correspondence: mary.jung@ubc.ca

${ }^{3}$ School of Health and Exercise Sciences Faculty of Health and Social

Development, The University of British Columbia Okanagan, RHS 1193333

University Way, Kelowna, BC V1V 1V7, Canada

Full list of author information is available at the end of the article
} 


\section{Background}

Levels of physical activity are assiduously low among children and youth in Canada [1] and worldwide [2], and have in part contributed to the increased rates of childhood overweight and obesity and associated chronic diseases (e.g., cardiovascular diseases and type 2 diabetes) [3, 4]. Establishing healthy lifestyle behaviours, like physical activity, is imperative during childhood, as these behaviours can extend across the life span [5] and have long-term health implications (e.g., prevention of weight gain/obesity, type 2 diabetes, coronary heart disease, dementia, Alzheimer's disease) [6]. Consequently, public health governing bodies have prioritized strategies and interventions to combat children's physical inactivity and obesity crisis globally [7, 8] and within Canada [9]. In Canada, schools are often the target of such initiatives as they represent an environment through which to reach a large and diverse population of youth, who spend a majority of their waking time in school $[10,11]$.

Several provinces in Canada have adopted daily physical activity policies aimed at increasing children's physical activity specifically during the school day [12-14]. In British Columbia, the Ministry of Education mandated a Daily Physical Activity (DPA) policy in 2008 (revised in 2011) requiring elementary schools to provide at least $30 \mathrm{~min}$ of DPA as part of the educational program for children in grades Kindergarten to seven [12]. Specifically, the DPA policy requires elementary students to achieve $30 \mathrm{~min}$ of physical activity at school on days with no physical education. ${ }^{1}$ This requirement includes any activities that help build endurance, strength and flexibility (e.g., walking, running, push-ups, stretching) and that are conducted during instructional (i.e. within-class) or non-instructional (i.e. recess or lunch break) time.

In order to improve the success of such policies, it is advised that policy evaluation occur at the outset and continues on an ongoing basis [15]; however, minimal research in Canada has examined the process of how DPA policy plans are translated into practice (i.e., implementation) and there is currently no research examining the effectiveness of the DPA policy in BC schools [16]. Central to understanding the implementation process is a comprehensive and theoretical examination of the numerous factors that can impede (i.e., barriers) or enhance (i.e., facilitators) the successful implementation of physical activity policies at a local school-level. While some research has identified barriers and facilitators to DPA implementation in Canada [17-21], theory is rarely used to guide our understanding of these factors [22]. Behaviour change theories postulate the psychological and environmental constructs that affect behaviour by specifying mechanisms of change. Within the school context, utilizing a theoretical approach allows researchers to systematically identify the potentially malleable factors affecting teacher's implementation of the policy and to prioritize and develop strategies through which to target these key factors to improve policy practice and achieve desired outcomes. For this reason, this study moves beyond the simple identification of barriers and facilitators to DPA policy implementation by descriptively linking these factors to pathways of behaviour change in order to enhance implementation practices [23].

To achieve this aim, this study uses the Theoretical Domains Framework (TDF). The TDF, developed and validated by Michie and colleagues, is an integrative framework that synthesizes over eighty constructs across thirty-three psychological theories in order to understand influences on behaviour more broadly [24, 25]. Specifically, the TDF is organized into 14 categories, called domains, to categorize the potential range of behavioural and organizational factors that influence implementation outcomes [26]. Domains that address behavioural factors include: Knowledge, Skills, Memory, attention and decision processes, Behavioural regulation, Social/professional role and identity, Beliefs about capabilities, Optimism, Beliefs about consequences, Intentions, Goals, Reinforcement, and Emotion. Domains that address organizational factors include: Environmental context and resources, and Social influences (TDF domain definitions are provided in Additional file 1).

The TDF has been successfully applied in many settings to identify influences on a variety of behaviours [27]. There are many individual, environmental and social-cultural factors that influence the successful implementation of policies in schools. For example, some of the factors shown to influence implementation include: leadership and support, resource support, communication/shared decisionmaking, and individual self-efficacy/skills [28]. Therefore, a framework that can capture these influences operating at different levels is warranted.

More broadly, the TDF is a refined version of the Capability Opportunity Motivation-Behaviour (COM-B) model, an evidence-based model supporting that three key sources (i.e., capability, opportunity and motivation) interact to influence behaviour. The COM-B model can be linked to a practical intervention design tool called the Behaviour Change Wheel framework (BCW) [26] to guide researchers in the selection of theory, intervention functions, policy categories, and behaviour change techniques for intervention design and delivery. As a result, the TDF is one of few frameworks linked to a comprehensive method for intervention design.

\section{Purpose}

The purpose of this study was to use the TDF to understand teachers' barriers and facilitators to the implementation of the Daily Physical Activity policy in British Columbia elementary schools. Additionally, barriers and facilitators were examined and compared according to how the teacher implemented the DPA policy during the school day (provision of DPA during instructional time or only non-instructional time). 


\section{Methods}

\section{Overall design}

This study used short surveys and semi-structured interviews to explore the factors (i.e., barriers and facilitators) associated with the implementation of the Daily Physical Activity policy by elementary school teachers in one school district in British Columbia. A content analysis was conducted using the TDF and overarching themes were identified within each domain. Ethical approval was obtained from the University of British Columbia's Behavioral Research Ethics Board for research involving humans, and the respective school district. The Consolidated criteria for reporting qualitative research (COREQ) [29] guided reporting of this study (see Additional file 2).

\section{Framework}

The first author used the TDF to construct the semistructured interview guide [see Additional file 3], which underwent revision by HG who is experienced in application of the TDF and was further refined after piloting the interview with two elementary school teachers. The TDF was then used to categorize the implementation barriers and facilitators and explore emergent themes by domain.

\section{Sample selection and recruitment}

One school district from British Columbia representing over 30 public elementary schools was chosen for convenience and approached to participate in this study. Principals of all elementary schools were emailed an information letter to request time to present the study to their intermediate teachers. The first investigator visited the school and conducted a presentation to the teachers, which consisted of information regarding the researcher's background and interests and her study purpose and details. Teachers were eligible to participate if they were grades 4,5 or 6 certified school teachers in publicly funded elementary schools with at least one year of experience teaching at an elementary school level, and were currently teaching in the 2015-2016 school year. In total, principals from 13 elementary schools (42\% response rate) provided approval for their school to participate, with 33 (of 40) teachers from 11 of these schools (83\% response rate) providing written consent to participate in a survey and potentially participate in the interview. The short survey instrument consisted of questions relating to the teacher's DPA implementation approaches and basic demographic information and was used as a device to assist in selecting and describing the interview sample. Based on survey responses, maximum variation sampling [30] was used to recruit teachers to be interviewed to ensure representation across teacher-reported implementation approaches, which continued until data saturation was reached [31]. In total, twelve interviews were conducted with thirteen teachers (4 male, 9 female), who were aged $30-60$ years $(M=44.69, S D=10.33)$ and varied in teaching experience from 5 to 34 years
$(M=15.69, S D=9.31)$. Of those teachers who were interviewed, one teacher taught grade 4 , three teachers taught grade $4 / 5$, two teachers taught grade 5 , five teachers taught grade $5 / 6$ and two teachers taught grade 6 . Ten teachers reported implementing DPA by providing additional opportunities to be active during instructional time (instructional implementers), while three teachers were classified as non-instructional implementers because they relied on students being active during non-instructional lunch and recess breaks.

\section{Data collection}

The first investigator conducted twelve semi-structured interviews with 13 teachers between February and April 2016, at a time and location convenient to each teacher (e.g., classroom, coffee shop). All interviews were conducted individually except for one interview, which included two grade 6 teachers from one school. The latter was done because these teachers share a formal platooning schedule (i.e., complete curriculum together within two classrooms), thus reporting the same DPA implementation approach. Each interview was between 31 and $64 \mathrm{~min}$ in duration $(M=52.25, S D=9.65)$ and consisted of a broad open-ended question (i.e., "Are there any factors that affect if or how you implement DPA in your classroom during class time? If so, what?") to elicit perceived barriers and facilitators impacting the implementation of the DPA policy by teachers. Probing questions were used to clarify domainspecific content if the participant had mentioned factors that appeared to fit within a certain domain (see Interview guide in Additional file 3 for more information). This approach was used to minimize leading questions. Field notes were taken by the interviewer during the interview to ensure each relevant domain was discussed further. Verbal consent was obtained from each participant to audiorecord the interview and participants received a monetary reimbursement $(\$ 20)$ for their participation.

\section{Data extraction and analysis}

Digital recordings were transcribed verbatim directly into NVivo Version 11 [32] by the first author and two research assistants. Interview transcripts were checked for accuracy by the interviewer; however, the transcripts were not returned to participants for comment. We employed a deductive and inductive analytical approach in a two-stage process whereby extracted barriers and facilitators were 1) deductively coded using pre-existing domains (content analysis based on TDF), and 2) analyzed for emergent themes within each domain. This analysis procedure was chosen because it provides a simple method for summarizing findings in the context of focused evaluation questions, while allowing exploration of unanticipated factors associated with implementation, and is commonly used in health research [33, 34] and TDF analyses [35-38]. 


\section{Barrier and facilitator extraction}

Barrier and facilitator extraction was performed by the first author, with double extraction occurring across $33 \%(n=4)$ of the transcripts by RM to ensure the trustworthiness of the data extraction and coding. Coders read through each interview transcript line-by-line, highlighting and coding the text to 'Barrier' or 'Facilitator' nodes (containers), operationalized as any factor, characteristic, view or belief that either impeded or enabled implementation of DPA by teachers during the instructional school day, respectively. Barriers and facilitators were extracted if the teacher being interviewed commented that the factor affected their own personal implementation of DPA or if they thought it affected other teachers' implementation of DPA (i.e., shadowed data). Hypothetical barriers and facilitators, characterized as a factor that the teacher perceived (versus experienced/encountered) to be a potential barrier or facilitator to them or other teachers, were not extracted (e.g., belief that specific resources or support would be helpful for implementation without past experience with these resources/support). If a teacher discussed the same barrier/facilitator at different times within the interview, the factor was counted as separate items. Therefore, the total frequency (count and percent) coded to each TDF domain represents the proportion of interview time spent discussing these factors within each domain. Discrepancies in extraction were discussed until a consensus was reached. Agreed upon barriers and facilitators were transferred to an Excel spreadsheet for TDF coding.

\section{Barrier and facilitator coding}

Two researchers independently coded barriers and facilitators from each interview over twelve rounds (each interview was a new round), with the order of each round being selected at random. As we were attempting to understand barriers and facilitators within the school context (and not test the reliability of the TDF), researchers coded in rounds and met to discuss discrepancies after each round. In the first round, identified barriers and facilitators were coded using the TDF domain and definitions as a coding framework (see Additional file 4) [26]. Where coding varied, consensus was achieved through discussion and the coding manual was refined for subsequent coding rounds to facilitate consistency of TDF coding (see 3rd column in Additional file 4). In the case of particularly challenging exerts, expertise was sought from an expert coder who is knowledgeable and experienced in application of the TDF. Coders also made notes and comments on the overall meaning of each exert during each coding round and responses were compared across teacherreported implementation approach type. The first coder identified main themes from each domain and exemplary quotations for each theme were selected, consistently crosschecking themes to original transcripts. Negative cases were highlighted and used to refine themes that accounted for the majority of cases. To confirm that interpretations were supported by the data, the themes were presented to the second coder and to an additional researcher who was not part of the data collection, extraction and coding for feedback.

\section{Reliability}

Percent agreement was used to show agreement on barrier and facilitator extraction. Percent agreements, Cohen's Kappa statistic [39] and prevalence-adjusted bias-adjusted Kappa statistic (i.e., PABAK) [40] were used to show agreement between coders on categorizing the barriers and facilitators by TDF domain, for new items coded at each round as well as for the overall total. PABAK represents the Kappa statistic that adjusts for 1) shared bias in the coders use of categories, and 2) the high prevalence of negative agreement (i.e., when both coders agree on non-contributing domains) and was used to account for the high prevalence of not assigning more than one domain to each barrier. Intercoder agreement values of $0.60-0.79$ indicate "substantial" reliability and those above 0.80 are "outstanding" [41].

\section{Results \\ Reliability}

The two independent coders extracted a total of 343 barriers/facilitators from four randomly selected interviews and percent agreement across all extraction rounds was 86.3\% (see Additional file 5). A total of 900 factors ( 417 barriers, 483 facilitators) were extracted across the twelve interviews. Upon coding, 68 (26 barriers, 42 facilitators) factors were deemed ineligible (due to being hypothetical or not affecting the targeted behaviour) and removed from the data set (see Additional file 6), leaving a total of 832 items. All items were coded into at least one of the fourteen TDF domains or an 'Other' category (for items that did not clearly fit into a pre-defined domain). Some items were coded to multiple TDF domains, resulting in a total of 1422 observations. Across all barrier and facilitator coding rounds, the average inter-coder agreement was outstanding (Percent agreement $=59.7 \%$; Kappa $=0.73 \pm 0.37$; PABAK $=0.91 \pm 0.13$ ). Overall reliability improved following refinement of the coding manual (see Additional file 7) and consensus of final codes was reached through discussion, resulting in 1141 final barrier and facilitator codes.

\section{Implementation barriers and facilitators}

Table 1 presents the summary of TDF domains, themes and quotes organized hierarchically by percent frequency for all participants. Accordingly, the most commonly coded TDF domains accounting for $75 \%$ of the total barriers and facilitators were Environmental context and resources (ECR; $n=250 ; 21.9 \%)$, Beliefs about consequences ( $n=225 ; 19.7 \%)$, Social influences $(n=193 ; 16.9 \%)$, Knowledge $(n=100$; $8.8 \%$ ), and Intentions ( $n=88 ; 7.7 \%)$. Only two items were classified as Other (or uncodable), due to a lack of specificity. Additional file 8 outlines the frequency (total count and 


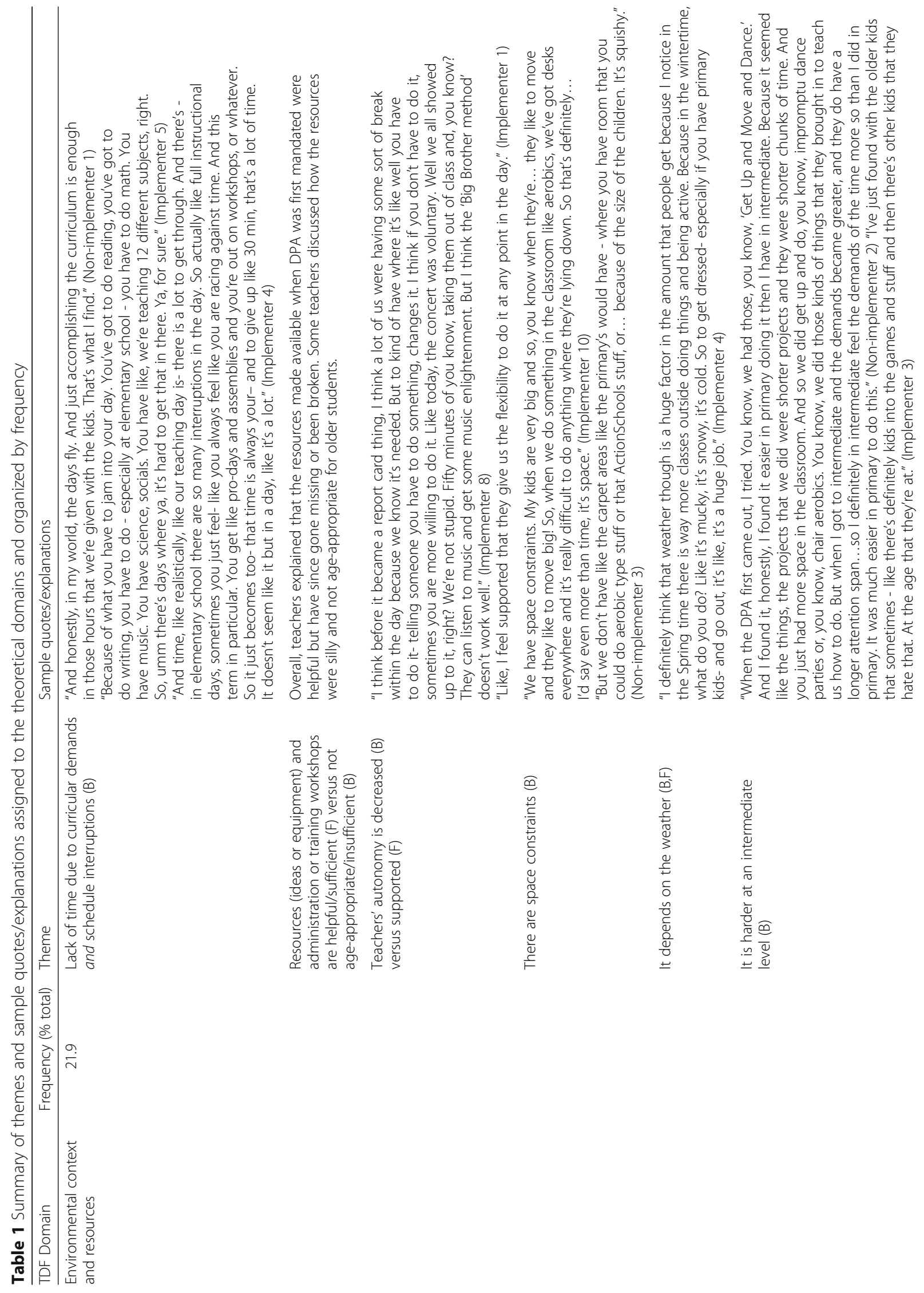




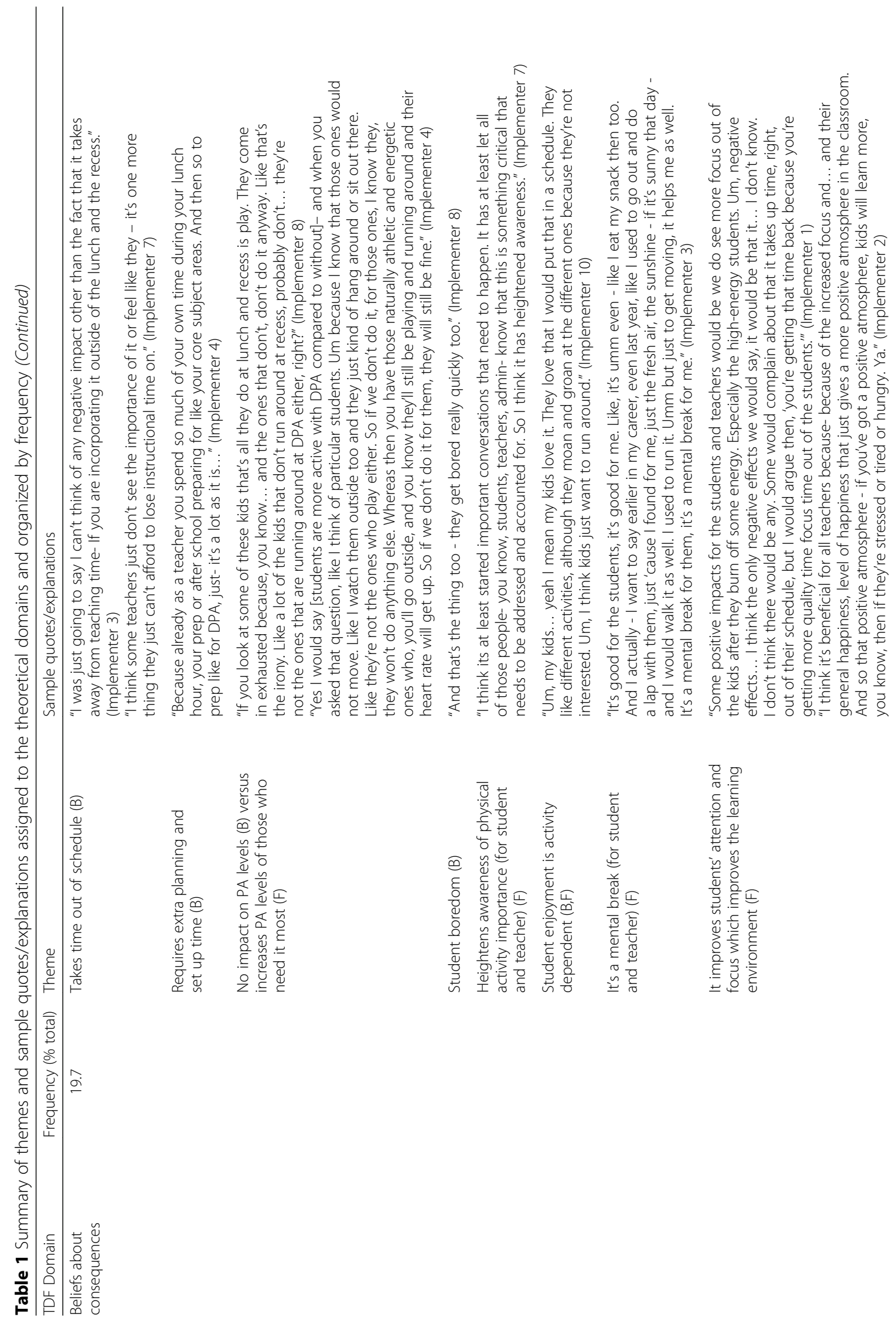




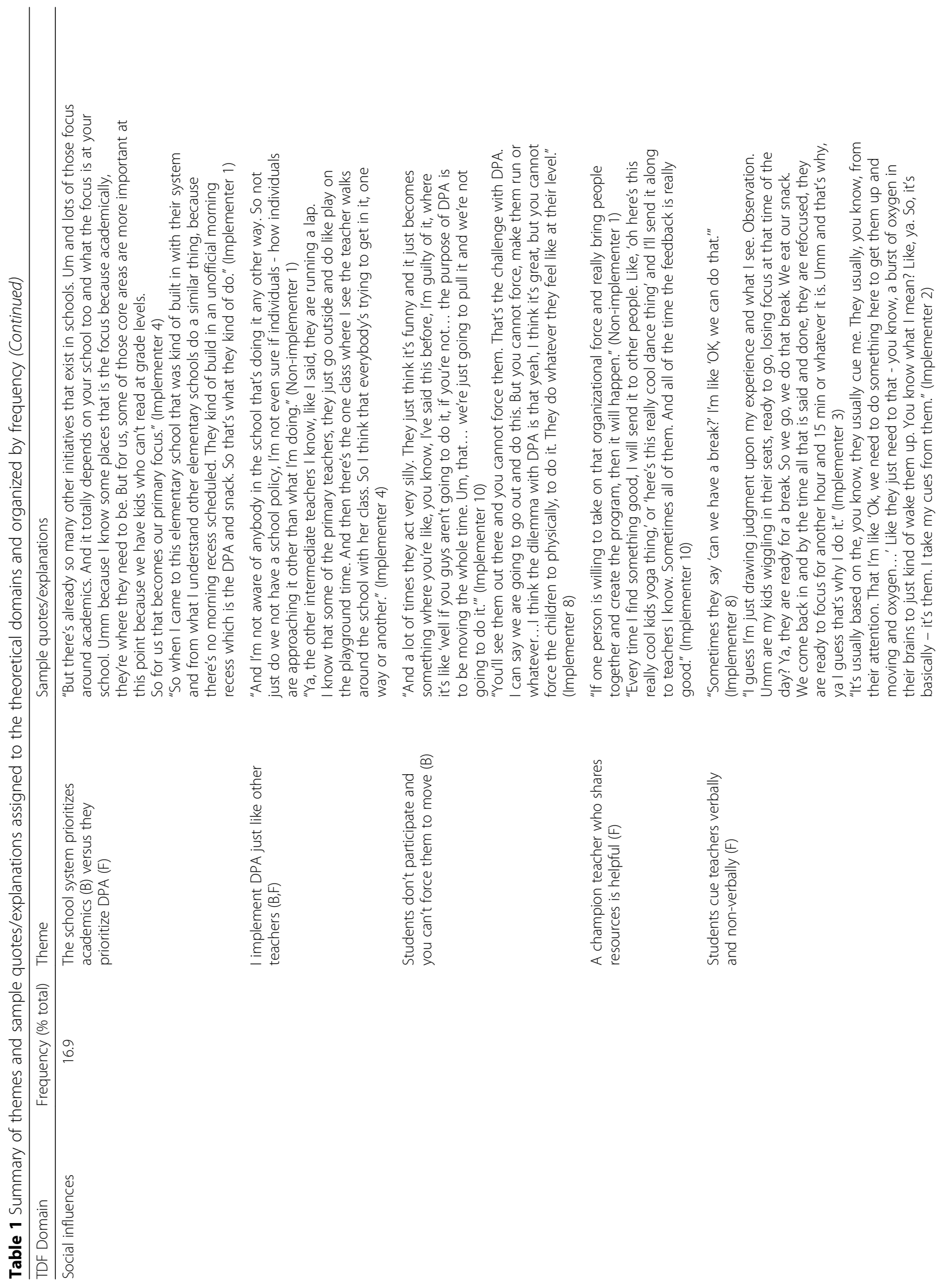




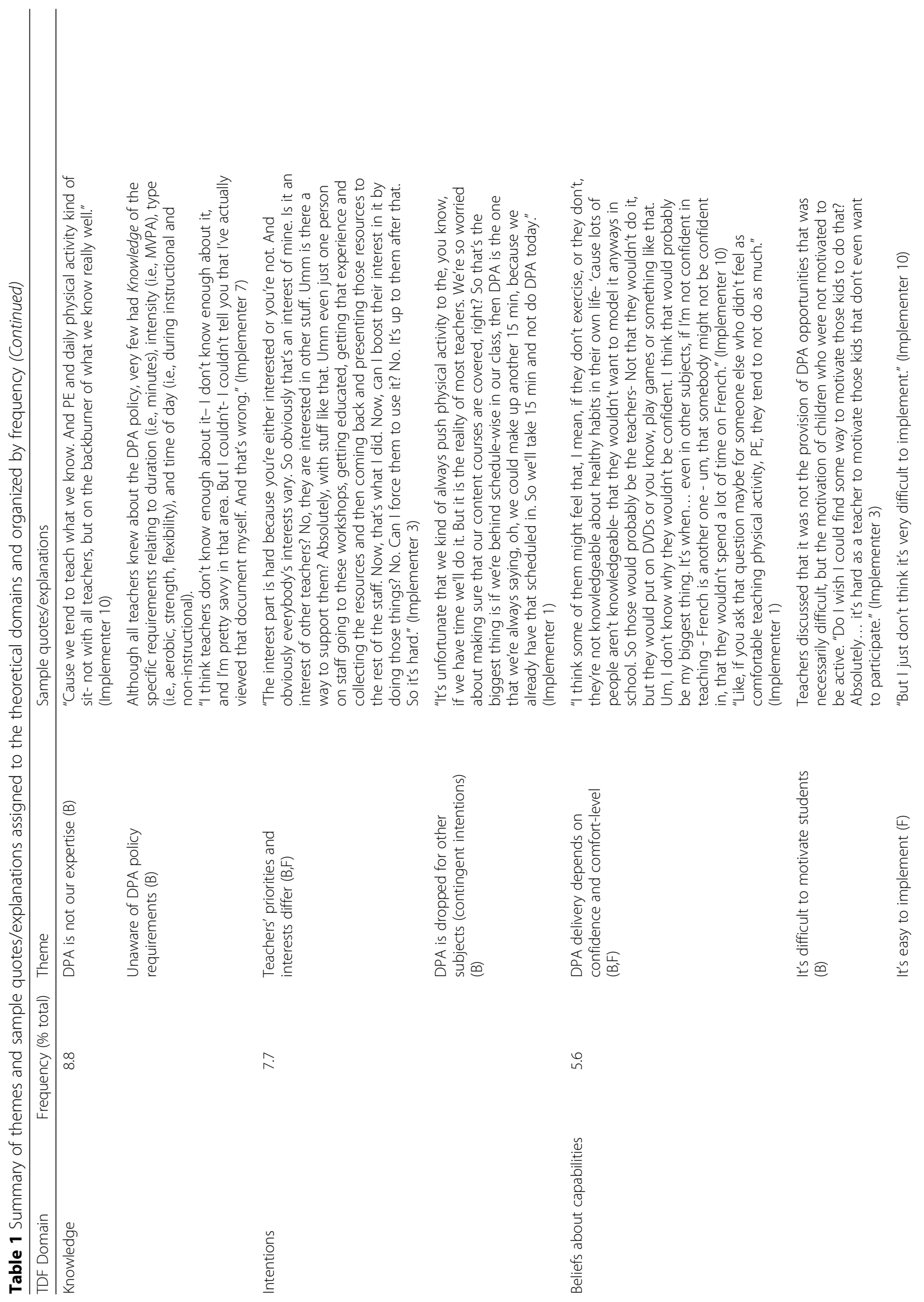




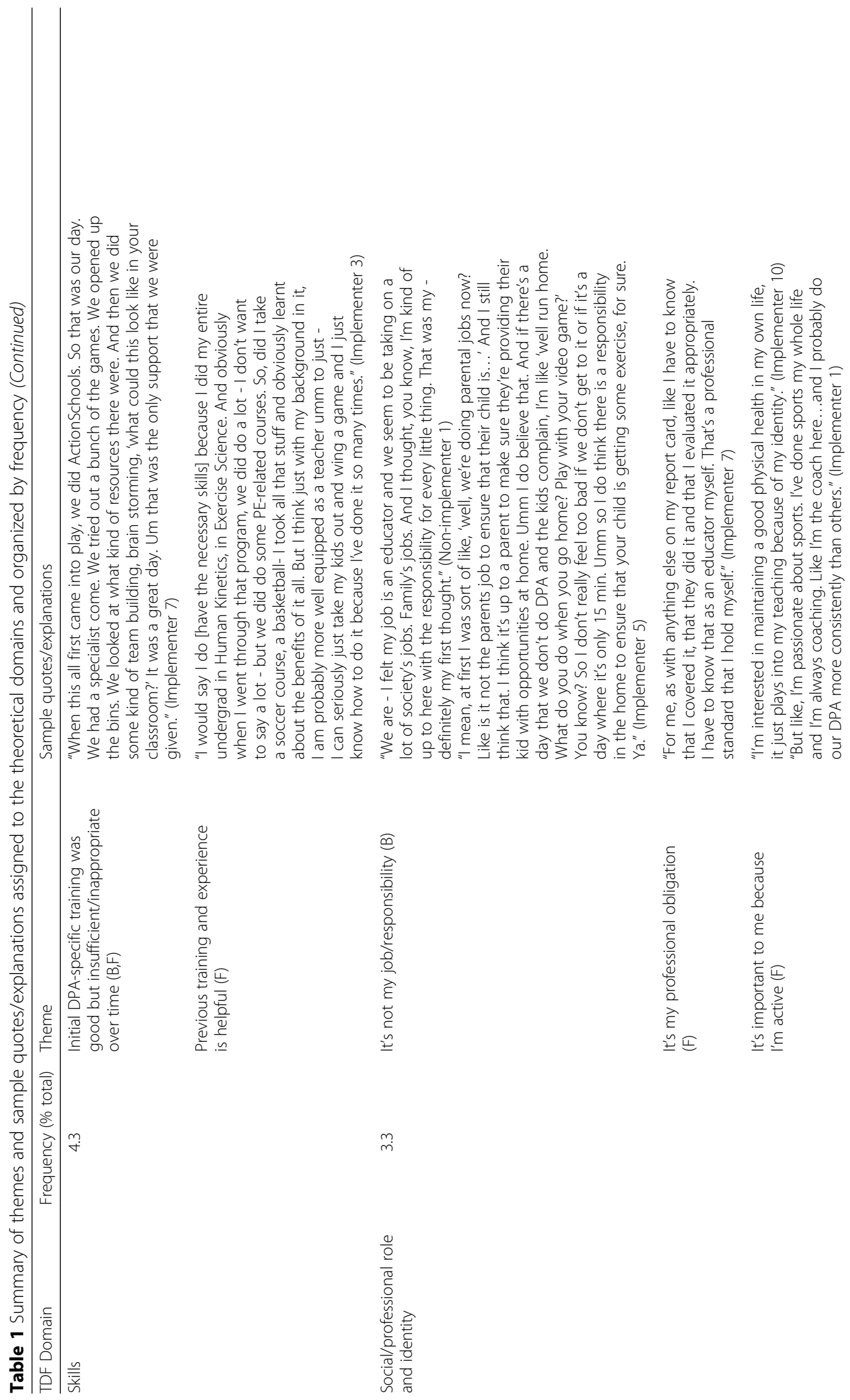




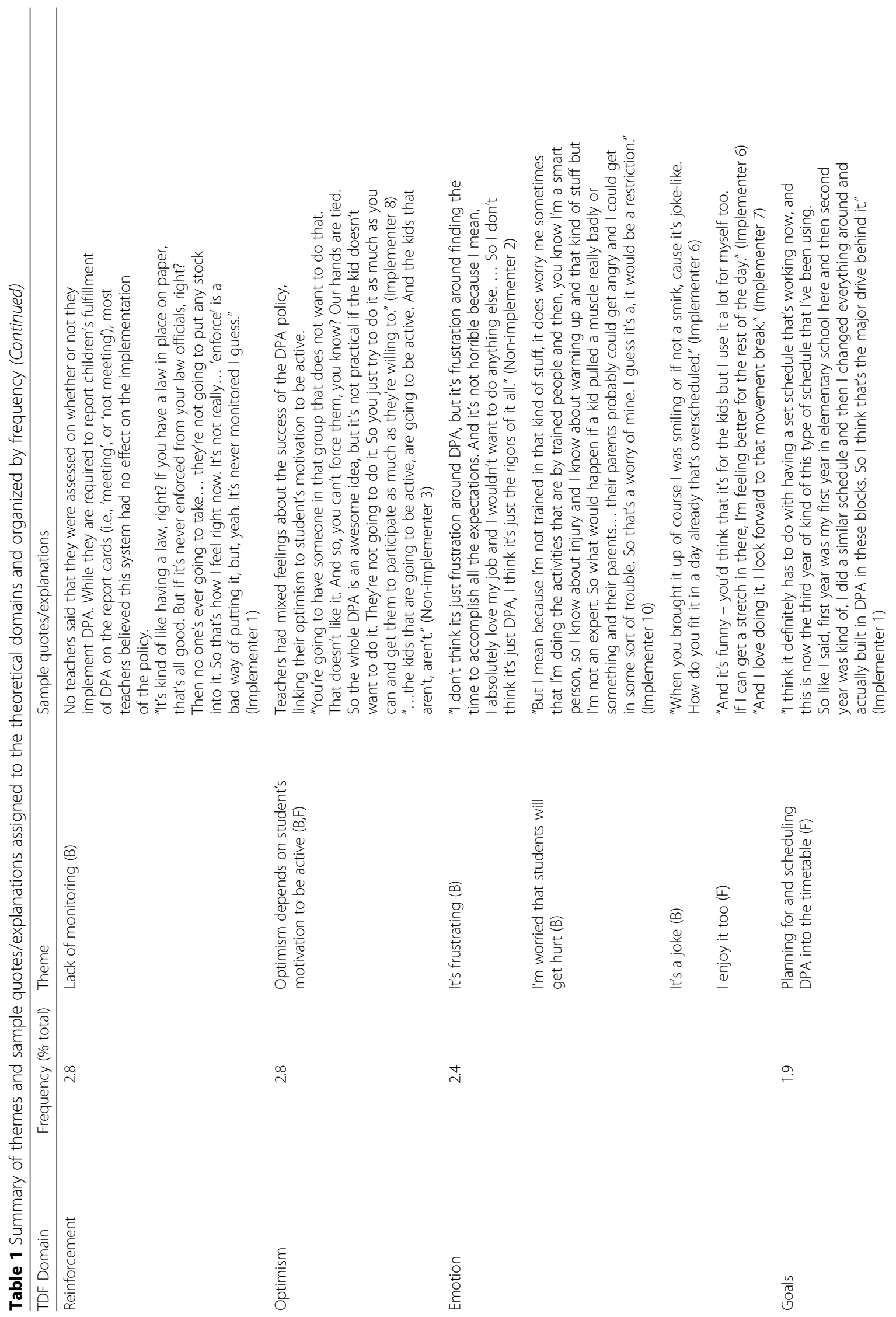




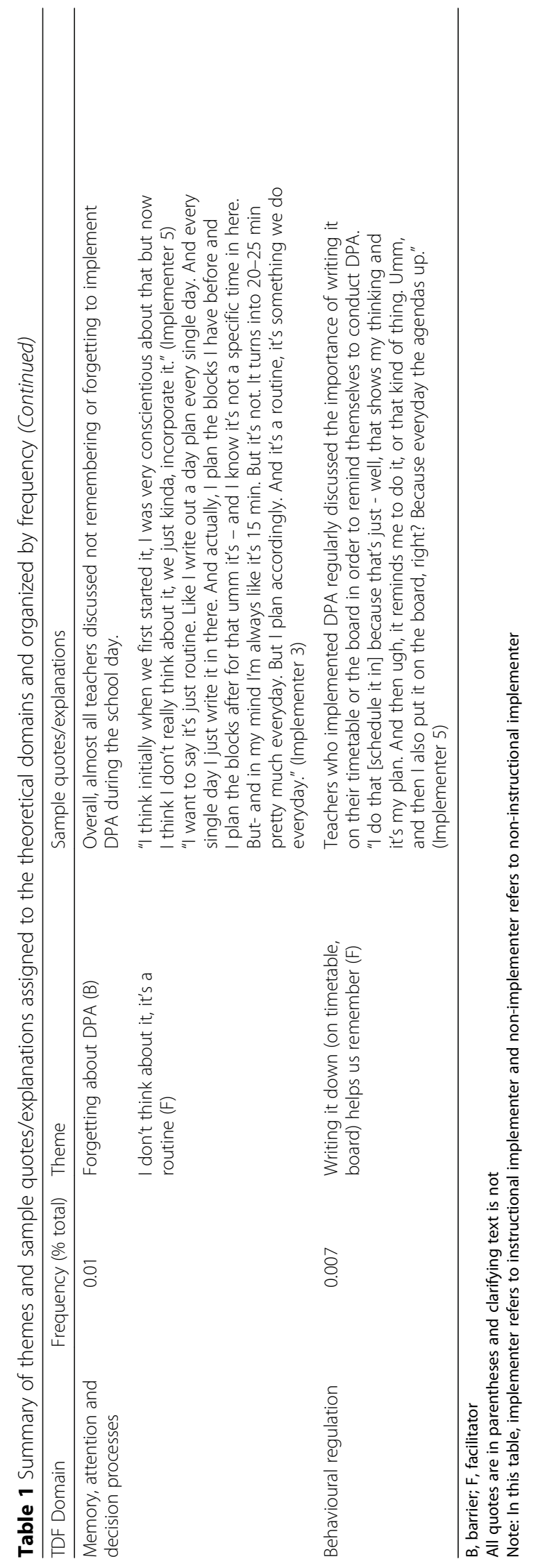


percent) of barriers/facilitators that were identified across each TDF domain by implementation approach group. Across all participants, more facilitators than barriers were discussed in relation to Knowledge, Behavioural regulation, Beliefs about consequences, Goals, and Social influences domains. Barriers and facilitators were equally discussed in Beliefs about capabilities, Optimism, and Intentions domains. Non-instructional implementers discussed rarely or not at all factors related to Memory, attention and decision processes, Behavioural regulation, and Goals. These differences are explored more descriptively in the next section.

\section{Comparison of barriers and facilitators by teacher implementation approach}

Teachers, irrespective of implementation approach (i.e., whether or not they provided DPA during the instructional school day) experienced similar barriers and facilitators with regards to Skills (e.g., DPA-specific training, previous training/experience), Knowledge (e.g., lack of knowledge about DPA requirements), Environmental context and resources (e.g., poor, inappropriate or lack of DPA-specific training; lack of time due to curricular demands and schedule interruptions; weather and space constraints), Reinforcement (e.g., lack of monitoring), Social influences (e.g., school-level priorities, support from other teachers, student participation), and Optimism (e.g., mixed feelings about success of policy).

Where teachers who implemented DPA during instructional time differed from those who relied on noninstructional time was in their Goals and Behavioural regulation (e.g., planning for and scheduling DPA in timetable; providing schedule to students), Social/professional role and identity (e.g., strong personal physical activity identity and belief in responsibility to get children active at school), experience of the Consequences (e.g., linking physical activity to improvements in attention and focus for a better classroom learning environment) and Social influences (e.g., recognizing and responding to children's verbal and non-verbal cues to move throughout the day).

\section{Discussion}

Similar to the review examining the barriers and facilitators to DPA policy implementation in Canada [42], this study highlights teachers' implementation of the DPA policy may be impacted by factors relating to ECR, Beliefs about consequences, and Social influences, as well as Knowledge and Intentions. The identified themes in this study have been reported in other DPA studies [17-21, 43-47], as well as studies examining the implementation of other school-based PA initiatives [48-52]. For example, similar themes in the ECR domain include lack of time in the schedule due to competing curricular demands [17$19,21,43-46,50-52]$, access to resources (space, facilities, equipment and ideas) [17-19, 21, 43-50], and inclement weather [43-46]. Related Beliefs about consequences themes include an increase in teacher workload, burden and stress $[18,44,46]$, improved student focus, attention and/or academic performance [17, 18, 44, 46, 49, 51], improved classroom learning environment [17-19, 46], and overall student enjoyment and interest in physical activity $[17,44,46]$. Similar themes within the Social influences domain include level of support from staff, administration and other school champions [18, 20, 21, 44, 48, 49], and student participation/preferences [18, 20, 21, 45, 48]. Due to these similarities, it is possible that intervention designs based on this study may be effective within other school contexts (e.g., different provinces/countries).

Addressing barriers to implementation is important because these factors affect implementation fidelity, which in turn has implications on the policy meeting its desired outcomes. Very little research has examined the impact of these policies on children's physical activity levels at school $[16,42]$. Considering the different approaches to implementation by teachers in this study, it is possible the different approaches result in different outcomes. This study compares similarities and differences in perceived barriers and facilitators to DPA implementation by teacher-reported implementation approach, suggesting that a targeted intervention approach is necessary for different contexts. Future studies should examine effectiveness of these approaches on physical activity levels of children at school through objective measurement. This study's findings can be used to provide context for and interpret why different DPA policy implementation approaches succeed or fail to meet intended outcomes at the student level [53].

While there are added challenges to the provision of DPA opportunities during instructional time (as opposed to relying on non-instructional time for children to be active), the instructional implementers were able to overcome these challenges. Common challenges reported by both instructional and non-instructional implementers included issues relating to $E C R$ (e.g., lack of time, resources and space) and Social influences (e.g., lack of school-level priority). It may be that teachers who implement DPA during instructional time are better able to overcome these underlying organizational barriers to DPA delivery. Accordingly, instructional teachers differed from non-instructional teachers on a number of behavioural domains, particularly those in which they could exert a degree of individual control, such as planning, scheduling and having strong personal beliefs in the importance of physical activity. For example, instructional implementers discussed facilitators with regards to Goals and Behavioural regulation (e.g., planning for and scheduling DPA in the timetable), and in their Social/professional role and identity (e.g., strong personal PA identity and belief in their responsibility to get children active at school). Non-instructional teachers did not plan for (i.e., set goals) or schedule DPA into their timetables (i.e., regulate their provision of DPA), both 
of which helped to facilitate instructional implementers provision of DPA opportunities during the instructional school day. Therefore, while it may seem that targeting barriers to DPA implementation may provide an effective means to improve implementation, an important distinction may be the factors that assist the instructional teachers in providing more DPA opportunities during instructional time. Researchers may want to consider these variations for intervention design and delivery in specific contexts.

\section{Implications}

The current study builds on previous research examining the factors influencing the implementation of DPA in Canada through the inclusion of an evidence-based determinant framework by which to provide a theory-based analysis of the implementation barriers and facilitators. Embedding these factors within the TDF domains enables researchers to develop interventions aimed at targeting the constructs shown to have the most salient influence on behaviour. This behavioural diagnosis is also relevant to policy makers who wish to better support teachers in their implementation efforts. In this study, teachers most often discussed factors within the ECR, Beliefs about consequences, Social influences, Knowledge and Intentions domains. When organized heuristically, these domains are representative of all sources of behaviour in the COM-B model, namely capability (Knowledge), opportunity (ECR, Social influences) and motivation (Beliefs about consequences, Intentions) components, and have important implications for theory selection in intervention design. These findings suggest that all components are interacting to influence teachers' DPA implementation behaviours, and therefore selecting a theory that broadly encompasses all determinants of behaviour may be more successful at promoting behavior change. Alternatively, reflective motivation theories may not be the most effective option for intervention design because they fail to consider the broader physical and social-environmental influences on behaviour. Findings from other DPA studies in Canada have found that both individual- and organizational-level factors influence DPA implementation. In Ontario, for example, Allison and colleagues [43] found that policy awareness, teacher self-efficacy, scheduling and monitoring are significant predictors of implementation fidelity. Efforts to improve implementation must target these individual- and system-level factors.

To create interventions, the relevant theoretical domains can be mapped onto intervention functions (e.g., via the Behaviour Change Wheel framework [BCW]) [26] and behaviour change techniques [23]. For example, possible intervention functions to target $E C R$ include Training, Restriction, Environmental restructuring and Enablement. To minimize teachers' perception of a lack of time (due to curricular demands), an intervention could be designed to train teachers how to incorporate physical activity into other lessons. Likewise, consideration of competing behaviours, namely other school curriculum subjects, may be another means by which to minimize the burden of a lack of time. As another example, the education intervention function could be used to target the teachers' lack of knowledge of DPA policy guidelines, and could be delivered by improving policy guideline dissemination and providing clear recommendations to teachers on how to achieve these guidelines.

After using the TDF to understand the behaviour, intervention designers can select the behaviour change techniques (BCTs), or active intervention components, aimed at targeting the relevant domains. For instance, BCTs that have been mapped to the ECR domain include: restructuring the physical or social environment, discriminative (learned) cue, prompts/cues, or avoidance/changing exposure to cues for the behaviour [54]. To address the lack of time example provided above, schools could restructure the environment by creating policies whereby teachers must schedule opportunities for their students to be active into their timetables. However, adoption of individual school policy would first require considerable changes to overcome factors working at the social- and structurallevel. Ultimately, final decisions about intervention functions, BCTs and modes of delivery can only be selected according to what can be feasibly and acceptably delivered within the specific school context [23].

\section{Strengths and limitations}

The main strength of this study was the use of the Theoretical Domains Framework to categorize and comprehend implementation barriers and facilitators. However, the TDF is not a theory, and therefore it cannot provide an explanation as to how these domains are connected and influence one another [53], limiting our understanding of how these factors interact in complex contexts. While the TDF showed good utility for categorizing barriers and facilitators within this context, it was difficult to differentiate between some domains (e.g., Beliefs about consequences and Optimism), noted too by other researchers [35, 37]. Additionally, using the TDF framework to guide the interview schedule and deductively code barriers and facilitators means that the researchers approached the data with an informed, yet potentially strong bias. However, the interview protocol was designed to minimize leading questions and extracting barriers and facilitators prior to coding into specific domains was done to minimize bias of identification of relevant text and increase trustworthiness. Although the interviewer asked participants to provide examples of barriers/facilitators that they had experienced versus perceived to impact DPA implementation, and efforts were made to minimize hypothetical barrier/facilitator extraction, it is possible that this distinction was not clearly discernable for 
participants. According to Sparkes and Smith [55], a general weakness of content analyses is that they suggest that the more themes or categories that are counted reflect the meaningfulness or significance of that category. In this study, the total count (n) coded to each TDF domain included repeated barriers/facilitators and each count reflect the proportion of time that the teachers dedicated to discuss the respective factor. Therefore, frequency of barriers/ facilitators coded to domains should not be a proxy for importance or significance. Some domains or themes that occurred only a few times may be highly meaningful to a teachers' implementation of DPA and thus be areas of potential interest (and future research) for those creating interventions to target these factors in the future. Finally, this study aimed to include teachers with diverse DPA implementation approaches. Unfortunately, it was difficult to identify and recruit teachers who did not provide DPA opportunities during instructional time, most likely due to social desirability bias.

\section{Conclusion}

Given that the effectiveness of school-based physical activity policies depends on their implementation, it is important to understand the challenges that teachers face in providing physical activity opportunities at school and to identify the levers that increase implementation. This study theoretically identified the barriers and facilitators impacting the implementation of the DPA policy in British Columbia and this information can be used to explain how the context influences the success or failure of the policy. The advantage of using a theoretical framework to understand the barriers is that it can assist researchers in the systematic development of future interventions to target the factors shown to impede implementation.

\section{Endnotes}

${ }^{1}$ Over the course of the planning for and data collection of this study, the BC Ministry of Education revised the DPA policy statement multiple times. As such, there is no longer any division between PE and non-PE days. The DPA requirements of $30 \mathrm{~min}$ of physical activity are now required irrespective of school days with or without PE.

\section{Additional files}

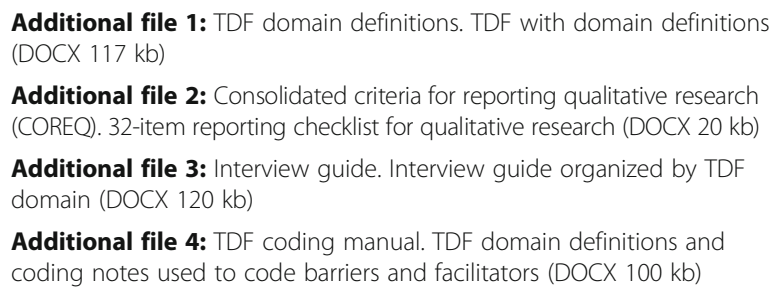

Additional file 5: Double extraction agreement. Inter-coder percent agreement across four barrier and facilitator extraction rounds (DOCX 40 kb)

Additional file 6: Ineligible extracted barriers and facilitators. Total counts of extracted barriers and facilitators that were ineligible or uncodable (DOCX 49 kb)

Additional file 7: Inter-coder agreement statistics. Inter-coder agreement statistics including percent agreement, Kappa and PABAK and the number of observations used during each coding round (DOCX $72 \mathrm{~kb}$ )

Additional file 8: Barriers and facilitators by TDF domain and implementation approach. Frequency counts of barriers and facilitators coded to each TDF domain by teacher implementation approach (DOCX $77 \mathrm{~kb})$

\begin{abstract}
Abbreviations
DPA: Daily Physical Activity policy; ECR: Environmental context and resources (TDF domain); PABAK: Prevalence adjusted bias adjusted Kappa statistic;

TDF: Theoretical Domains Framework
\end{abstract}

\section{Acknowledgements}

We would like to thank the school district and participants for their time and responses provided in the interviews.

\section{Funding}

KW received funding from the Canadian Institutes of Health Research - Canada Graduate Scholarship to conduct this research and the project was funded by a Michael Smith Foundation for Health Research grant (\#5917) to MJ.

\section{Availability of data and materials}

The datasets generated and/or analysed during the current study are not publicly available in order to maintain participant privacy.

\section{Authors' contributions}

KW conceptualized the study, and MJ/HG provided intellectual input into the methodological design. KW conducted the interviews, transcribed and analyzed the interview data and drafted the manuscript. RM provided double extraction and coding of implementation barriers and facilitators. All authors reviewed and approved the final manuscript.

\section{Ethics approval and consent to participate}

This research was performed in accordance with the Declaration of Helsinki and ethical approval was obtained from the Canadian University's Behavioural Research Ethics Board for research involving humans, and the respective school district. All participants provided written informed consent.

Consent for publication

Not applicable.

Competing interests

The authors declare that they have no competing interests.

\section{Publisher's Note}

Springer Nature remains neutral with regard to jurisdictional claims in published maps and institutional affiliations.

\section{Author details}

${ }^{1}$ School of Health and Exercise Sciences | Faculty of Health and Social Development, The University of British Columbia Okanagan, ART 360- 1147 Research Road, Kelowna, BC V1V 1V7, Canada. ${ }^{2}$ School of Health and Exercise Sciences Faculty of Health and Social Development, The University of British Columbia Okanagan, ART 129- 1147 Research Road, Kelowna, BC V1V 1V7, Canada. ${ }^{3}$ School of Health and Exercise Sciences Faculty of Health and Social Development, The University of British Columbia Okanagan, RHS 1193333 University Way, Kelowna, BC V1V 1V7, Canada. 
Received: 15 March 2017 Accepted: 13 October 2017

Published online: 23 October 2017

\section{References}

1. ParticipACTION. Are Canadian kids too tired to move? The 2016 ParticipACTION report card on physical activity for children and youth. Toronto: ParticipACTION; 2016. https://wwwparticipactioncom/sites/default/ files/downloads/2016\%20ParticipACTION\%20Report\%20Card\%20\%20Full\%20Reportpdf. Accessed 2 Dec 2016.

2. Roberts C, Tynjälä J, Komkov A. Physical activity. In: young people's health in context. Health behaviour in school-aged children (HBSC) study: international report from the 2001/2002 survey. World health. Organization. 2004. http://www.euro.who.int/_data/assets/pdf_file/0008/110231/e82923. pdf. Accessed 2 Dec 2016.

3. Ball GDC, McCargar LJ. Childhood obesity in Canada: a review of prevalence estimates and risk factors for cardiovascular diseases and type 2 diabetes. Can J Appl Physiol. 2003;28:117-40

4. Tremblay MS, Willms JDI. The Canadian childhood obesity epidemic related to physical inactivity? Int J Obes Relat Metab Disord. 2003;27:1100-5.

5. Telama R. Tracking of physical activity from childhood to adulthood: a review. Obes Facts. 2009;3:187-95.

6. Reiner M, Niermann C, Jekauc D, Woll A. Long-term health benefits of physical activity - a systematic review of longitudinal studies. BMC Public Health. 2013;13:813.

7. World Health Organization: Global recommendations on physical activity for health. 2010. http://www.who.int/dietphysicalactivity/factsheet recommendations/en/. Accessed 21 Nov 2016.

8. Waters E. De Silva-Sanigorski a, Burford BJ, Brown T, Campbell KJ, Gao Y, et al. interventions for preventing obesity in children. Cochrane Database Syst Rev. 2011. https://doi.org/10.1002/14651858.CD001871.pub3.

9. Taylor JP, McKenna ML, Butler GP. Monitoring and evaluating schools nutrition and physical activity policies. Can J Public Health. 2010;101:S24-7.

10. Lagarde F, LeBlanc CMA. Policy options to support physical activity in schools. Can J Public Health. 2010;101:S9-S13.

11. Naylor PJ, McKay HA. Prevention in the first place: schools a setting for action on physical inactivity. Br J Sports Med. 2009;43:10-3.

12. British Columbia Ministry of Education: Daily physical activity. 2016. https:// www.bced.gov.bc.ca/dpa/dpa_requirement.htm. Accessed 24 Oct 2016.

13. Alberta Education: Daily physical activity initiative. 2016. http://education. alberta.ca/teachers/resources/dpa.aspx. Accessed 24 Oct 2016.

14. Public Health Ontario: Daily physical activity in Ontario. 2016. http:/www publichealthontario.ca/en/BrowseByTopic/HealthPromotion/Pages/Daily-PhysicalActivity-in-Ontario.aspx\#.VQZZNmY2m2w. Accessed 24 Oct 2016.

15. Ramanthan S, Allison KR, Faulkner G, Dwyer JJM. Challenges in assessing the implementation and effectiveness of physical activity and nutrition policy interventions as natural experiments. Health Promot Int. 2008;23:290-7.

16. Olstad DL, Campbell EJ, Raine KD, Nykiforuk CIJA. Multiple case history and systematic review of adoption, diffusion, implementation and impact of provincial daily physical activity policies in Canadian schools. BMC Public Health. 2015;15:385-426.

17. Alberta Education: Daily physical activity survey report. 2008. https:/education. alberta.ca/media/160221/dpasurveyreport.pdf. Accessed 4 Dec 2015.

18. Brown KM, Elliott SJ. It's not as easy as just saying 20 minutes a day': exploring teacher and principal experiences implementing a provincial physical activity policy. Univers J Public Health. 2015;3:71-83.

19. Patton I. Teacher's perspectives of the daily physical activity program in Ontario. Phys Health Ed J. 2012;78:14-21.

20. Rickwood G. The status of daily physical activity in northern Ontario's elementary public schools. J Educ Train Stud. 2015;3:136-49.

21. Strampel CM, Martin L, Johnson MJ, lancu HD, Babineau C, Carpenter JG. Teacher perceived barriers and potential solutions to implementing daily physical activity in elementary schools. Phys Health Ed J. 2014;80:14-22.

22. Rothman AJ. "Is there nothing more practical than a good theory?": why innovations an advances in health behaviour change will arise if interventions are used to test and refine theory. Int J Behav Nutr Phys Act. 2004;1:11-7.

23. French SD, Green SE, O'Connor DA, McKenzie JE, Francis JJ, Michie S, et al. Developing theory-informed behaviour change interventions to implement evidence into practice: a systematic approach using the theoretical domains framework. Implement Sci. 2012;7:38-45.
24. Cane J, O'Connor D, Michie S. Validation of the theoretical domains framework for use in behaviour change and implementation research. Implement Sci. 2012;7:37.

25. Michie S, Johnston M, Abraham C, Lawton R, Parker D, Walker A. Making psychological theory useful for implementing evidence based practice: a consensus approach. Qual Safe Health Care. 2005;14:26-33.

26. Michie S, Atkins L, West R. The behaviour change wheel: a guide to designing interventions. Great Britain, UK: Silverback Publishing; 2014.

27. Atkins L, Francis J, Islam R, O'Connor D, Patey A, Ivers N, Michie SA. Guide to using the theoretical domains framework of behaviour change to investigate implementation problems. Implement Sci. 2017;12:77.

28. Clarke AM, O'Sullivan M, Barry MM. Context matters in programme implementation. Health Educ. 2010;110(4):273-93.

29. Tong A, Sainsbury P, Craig J. Consolidated criteria for reporting qualitative research (COREQ): a 32-item checklist for interviews and focus groups. Int J Qual Health Care. 2007;19:349-57. https://doi.org/10.1093/intqhc/mzm042.

30. Onwuegbuzie AJ, Collins KMTA. Typology of mixed methods sampling designs in social science research. Qual Rep. 2007;12:281-316

31. Richards L, Morse JM. Readme first for a user's guide to qualitative methods ( $3^{\text {rd }}$ ed.). Thousand Oaks, CA: SAGE Publications, Inc; 2013.

32. NVivo qualitative data analysis software (Version 11). Australia. QSR international Pty. In: Ltd; 2016.

33. Hsieh HF, Shannon SE. Three approaches to qualitative content analysis. Qual Health Res. 2005;15:1277-88.

34. Thomas DRA. General inductive approach for analyzing qualitative evaluation data. Am J Eval. 2006;27:237-46.

35. Heslehurst N, Newham J, Maniatopoulos G, Fleetwood C, Robalino S, Rankin J. Implementation of pregnancy weight management and obesity guidelines: a meta-synthesis of healthcare professionals' barriers and facilitators using the theoretical domains framework: implementing pregnancy weight guidelines. Obes Rev. 2014;15:462-86.

36. Lawton R, Heyhoe J, Louch G, Ingleson E, Willis TA, McEachan RRC, Foy R. Using the theoretical domains framework (TDF) to understand adherence to multiple evidence-based indicators in primary care: a qualitative study. Implement Sci. 2016;11:113.

37. Phillips CJ, Marshall AP, Chaves NJ, Jankelowitz SK, Lin IB, Loy CT, et al. Experiences of using the theoretical domains framework across diverse clinical environments: a qualitative study. J Multidiscip Healthc. 2015:8:139-46.

38. Patey AM, Islam R, Francis JJ, Bryson GL, Grimshaw JM, Canada PPT. Anesthesiologists' and surgeons' perceptions about routine pre-operative testing in low-risk patients: application of the theoretical domains framework (TDF) to identify factors that influence physicians' decisions to order pre-operative tests. Implement Sci. 2012;7:52

39. Cohen J. Weighted kappa: nominal scale agreement provision for scaled disagreement or partial credit. Psychol Bull. 1968;70:213-20.

40. Byrt T, Bishop J, Carlin J. Bias, prevalence and kappa. J Clin Epidemiol. 1993; 46:423-9.

41. Landis JR, Koch GG. The measurement of observer agreement for categorical data. Biometrics. 1977:33:159-74.

42. Weatherson KA, Gainforth HL, Jung ME. A Theoretical Analysis of the Barriers and Facilitators to the Implementation of School-Based Physical Activity Policies in Canada: A Mixed Methods Scoping Review. Implement Sci. 2017;12(1):41.

43. Allison KR, Vu-Nguyen K, Ng B, Schoueri-Mychasiw N, Dwyer JJM, Manson H, et al. Evaluation of Daily Physical Activity (DPA) policy implementation in Ontario: surveys of elementary school administrators and teachers. BMC Public Health. 2016;16:746.

44. Allison KR, Schoueri-Mychasiw N, Roberts J, Hobin E, Dwyer JJ, Manson H. Development and implementation of the Daily Physical Activity policy in Ontario, Canada: a retrospective analysis. Revue phénEPS/PHEnex J. 2014:6:1-18.

45. Kennedy CD, Cantell M, Dewey D. Has the Alberta daily physical activity initiative been successfully implemented in Calgary schools? Paediatr Child Health. 2010;15:e19-e24.

46. Mâsse LC, Naiman D, Naylor PJ. From policy to practice: implementation of physical activity and food policies in schools. Int J Behav Nutr Phys Act. 2013;10:71-82.

47. Robertson-Wilson JE, Lévesque L. Ontario's Daily Physical Activity policy for elementary schools: is everything in place for success? Can J Public Health. 2009;100:125-129.

48. Hatfield DP, Chomitz VR. Increasing children's physical activity during the school day. Curr Obes Rep. 2015;4:147-156. 
49. Naylor PJ, Nettlefold L, Race D, Hoy C, Ashe MC, Higgins JW, McKay HA. Implementation of school based physical activity interventions: a systematic review. Prev Med. 2015;72:95-115.

50. Dwyer JJM, Allison KR, Barrera M, Hansen B, Goldenberg E, Boutilier MA. Teachers' perspective on barriers to implementing physical activity curriculum guidelines for school children in Toronto. Can J Public Health. 2003;94:448-452

51. Evenson KR, Ballard K, Lee G, Ammerman A. Implementation of a schoolbased state policy to increase physical activity. J Sch Health. 2009;79:231-238

52. Larsen T, Samdal O, Tjomsland H. Physical activity in schools: a qualitative case study of eight Norwegian schools' experiences with the implementation of a national policy. Health Educ. 2012;113:52-63.

53. Nilson P. Making sense of implementation theories, models and frameworks. Implement Sci. 2015;10:53.

54. Cane J, Richardson M, Johnston M, Ladha R, Michie S. From lists of behaviour change techniques (BCTs) to structured hierarchies: comparison of two methods of developing a hierarchy of BCTs. Br J Health Psychol. 2015;20:130-150

55. Sparkes AC, Smith B. Qualitative research methods in sport, exercise \& health: from process to product. New York, NY: Routledge; 2014.

Submit your next manuscript to BioMed Central and we will help you at every step:

- We accept pre-submission inquiries

- Our selector tool helps you to find the most relevant journal

- We provide round the clock customer support

- Convenient online submission

- Thorough peer review

- Inclusion in PubMed and all major indexing services

- Maximum visibility for your research

Submit your manuscript at www.biomedcentral.com/submit
Biomed Central 